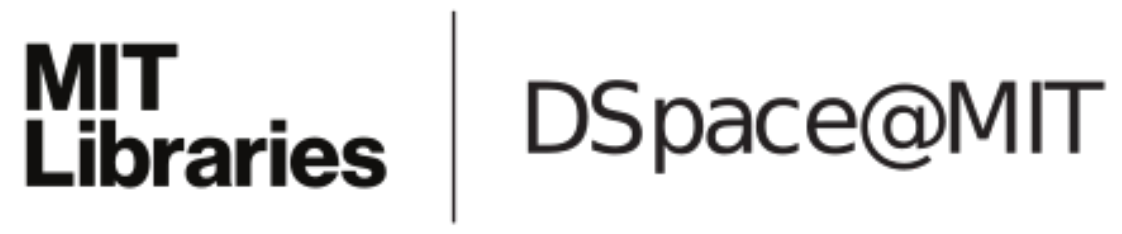

\author{
MIT Open Access Articles
}

Vehicular engine oil service life characterization using On-Board Diagnostic (OBD) sensor data

The MIT Faculty has made this article openly available. Please share how this access benefits you. Your story matters.

Citation: Siegel, J., et al. “Vehicular Engine Oil Service Life Characterization Using On-Board Diagnostic (OBD) Sensor Data." IEEE SENSORS 2014 Proceedings, IEEE, 2014, pp. 1722-25.

As Published: http://dx.doi.org/10.1109/ICSENS.2014.6985355

Publisher: Institute of Electrical and Electronics Engineers (IEEE)

Persistent URL: http://hdl.handle.net/1721.1/117390

Version: Author's final manuscript: final author's manuscript post peer review, without publisher's formatting or copy editing

Terms of use: Creative Commons Attribution-Noncommercial-Share Alike 


\title{
Vehicular Engine Oil Service Life Characterization Using On-Board Diagnostic (OBD) Sensor Data
}

\author{
J. Siegel*, R. Bhattacharyya*, A. Deshpande*†, S. Sarma* \\ Email: j_siegel, rahul_b and sesarma@mit.edu, ajayd@us.ibm.com \\ * Department of Mechanical Engineering \\ Massachusetts Institute of Technology \\ Cambridge, MA 02139 \\ ${ }^{\dagger}$ IBM Research \\ Yorktown Heights, NY 10598
}

\begin{abstract}
Standardized vehicular On-Board Diagnostics (OBD) systems offer access to information commonly used for fault notification and reactive diagnostic services. Recently, there have been efforts to use OBD data to diagnose and predict faults prior to catastrophic failure events. Engine oil service life, a parameter directly related to engine longevity, is difficult to measure conventionally. We show that the rate of engine coolant temperature rise, readily obtainable through the OBD suite, can serve as a proxy to indicate the remaining engine oil life. We demonstrate consistent results for one vehicle under similar environmental and unloaded engine operating conditions. We also examine the validity of this approach under varying environmental and engine loading conditions with tests on a second vehicle.
\end{abstract}

\section{INTRODUCTION}

Proper engine lubrication is critical to the safe and efficient operation of internal combustion engines. The constant heating, churning, and contamination related to engine use thickens oil, increasing drag on rotating assemblies. Drag and heat generation have a significant impact on engine performance, fuel economy, and rotating assembly longevity. Thickened, overheated oil may restrict flow to or entirely block small clearance engine passages, causing cascading lubrication and thermal issues. Short oil change intervals reduce the likelihood of catastrophic engine failure and keep combustion engines in excellent operating condition, avoiding a growing problem as the average age of vehicles in operation increases. ${ }^{1}$ Optimal timing of oil changes therefore minimizes vehicle cost of ownership and maximizes reliability and performance.

There are two approaches to monitoring engine oil viscosity today. One approach is to use embedded sensors for in situ measurement of engine oil viscosity. Sensors that make use of acoustic, electrical, chemical or direct viscosity measurements are commercially available today [1], [2], [3]. However, the deployment of such sensors is limited in practice due to cost, complexity, and singular utility [4].

As a lower-cost alternative, many automotive OEMs use proprietary algorithms counting engine revolutions or otherwise creating an oil life metric as the function of engine load, vehicle speed, and operational time, among other inputs. These algorithms, and others discussed in public patent filings, are fed information from the conventional On-Board Diagnostic network, and occasionally incorporate proprietary sensor information beyond that included with standard diagnostic parameters, such as oil pressure. Cloud-based tools such as OnStar have enabled real time access to OEM diagnostic data and heuristics [5]. However, such algorithms duplicate in situ results or are open-loop, inferring oil life based on historic data and rarely or never validating accuracy. These algorithms often overestimate engine oil change frequency, especially when paired with modern synthetic engine oils having improved wear resistance. The error factors in open-loop models are substantial, causing manufacturers and users alike to err on the side of requiring changes more frequently, but even in such cases these corrected models are prone to underestimation when subjected to extreme engine loading or poor air filtration. Limited data availability forces older vehicles to simply make use of distance and possibly time since last oil change to infer lubricant aging based on empirical and experiential models.

The On-Board Diagnostics II (OBDII) system, built into US car models on and after 1996, is capable of providing realtime information from emissions-related sensors, such as engine speed, coolant temperature, fuel trim, and timing advance, as well as 'freeze frame' data and trouble codes [6]. Similar standards exist internationally as well [7]. Unfortunately, these specifications do not require the measurement of any combustion engine oil parameters, so are not directly useful in quantifying remaining lubricant life. Despite the limitation of OBD information applicability, some standardized values may inform applications about the broader operating state of the vehicle. As an example, researchers have made use of OBD velocity data to improve the trajectory estimate of a vehicle relative to GPS [8]. In this paper, we make use of the fact that oil status manifests in several aspects of engine operation. Specifically we examine if the rate of rise of engine coolant temperature, an available OBDII parameter, can be reliably used as a proxy for remaining oil life. This technique relies on indirect measurement to observe the vehicle's operation and infer the state of oil health based on telltale indicators.

\footnotetext{
${ }^{1}$ Based on a 2013 Polk study
} 


\section{EXPERIMENTAL HYPOTHESIS}

As engine oil thermally degrades and picks up soot and other contaminants, the effective viscosity increases. This raised viscosity increases friction and heat generation in the engine due to heightened shear stresses and resultant viscous heating, as oil is continually forced through constant-width pathways inside the engine and used to lubricate pistons and cylinders with high relative velocities and narrow clearances. Steadystate engine operating temperature is unrelated to oil thickness, as engine thermostats tend to regulate maximum engine temperature to a high degree of precision. Despite this control system, there is another thermal metric that provides insight into the engine operating characteristics. A simple coolant temperature sensor found in all OBDII-compliant vehicles may be used to infer engine oil health. This is because the engine coolant temperature closely follows the temperature of the engine and oil (until the moment when the thermostat opens to regulate coolant temperature) and because heat generation is dominated by friction, a factor moderated by oil viscosity.

An engine with newly filled oil will have lower friction and heat up more slowly than an engine with oil near end-of-life. An engine with old oil will have increased friction due to higher effective viscosity and flow resistance. The derivative of the engine coolant temperature $\left(T_{\text {cool }}\right)$ with respect to time, i.e. $\frac{d T_{\text {cool }}}{d t}$, will thus increase as oil wears out. This allows the indirect measurement of remaining oil life, which will therefore indicate the state of wear for oil in an engine and provide closed-loop feedback, improving oil change recommendations. In effect, the coolant temperature becomes a surrogate metric for engine oil viscosity, which when tracked over time can highlight degradation based on open access data.

\section{Logging System Architecture}

Two data logging systems were used for data collection. The first system was a direct-to-Cloud data capture system, while the second system was a standalone logging device. Both systems captured OBD information and GPS information for later analysis on remote computing systems.

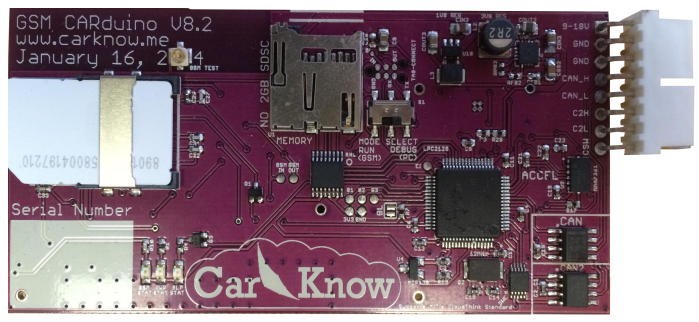

Fig. 1. The CARduino device connects to a standardized diagnostic connector and captures information from On-Board Diagnostic sensors, CAN signals, GPS, accelerometers, and gyroscopes.

Initial testing results were recorded with the use of a purpose-built telemetry device called the CarKnow CARduino, shown in Figure 1. The CARduino was selected because it provides an easy to configure car-to-Cloud interface and multiple data capture sources, including raw Controller Area
Network (CAN) access. In testing, this device was used to capture only OBDII sensor data from the in-vehicle CAN at regular intervals, along with GPS coordinates for timestamping and weather validation. Sensor values were then transmitted via GSM to a Cloud server for data capture and storage (Figure 2). ${ }^{2}$ The CloudThink RESTful API provided access to a digitally duplicated vehicle object (an 'Avacar') containing raw sensor data for post-processing, made available in JSON and plaintext formats [9].

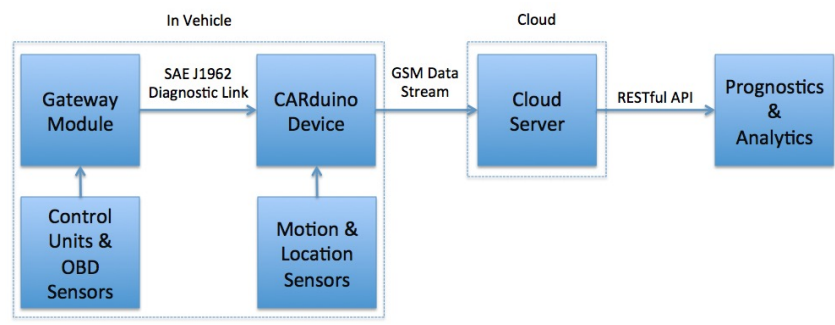

Fig. 2. Data was collected from the in-vehicle Controller Area Network with a CARduino diagnostic device, transmitted to a Cloud server, and the results served via a RESTful API.

The CARduino devices were pivotal in collecting data for controlled experimentation, but as a result of limited hardware supply, subsequent testing relied on the use more broadly available devices. The module included in the experiment was the DashDyno by Auterra [10], which logged data to a memory card capable of conversion to comma-separated values.

\section{EXPERIMENTAL SETUP}

The initial experimental procedure eliminated environmental factors including ambient temperature, airflow, and driver behavior. Tests were performed on a stock 2011 Buick Regal with a 2.0 liter turbocharged engine. Engine oil before and after testing was identical DEXOS-approved major brand $5 \mathrm{~W}$ 30 synthetic motor oil, as recommended by the manufacturer. Follow-up testing was performed on a 2003 Toyota Camry, where the driver was instructed to use the vehicle as he typically would. The uncontrolled experiment added complicating factors such as changing engine loading and airflow to the model in an attempt to illustrate the impact such factors have on oil temperature changes.

\section{A. Controlled Warmup}

The basic test procedure involved allowing the engine to cool down for a minimum of two hours since the last start to provide a sufficiently low starting temperature, connecting diagnostic hardware, and configuring the device to begin logging engine coolant temperature at $1 \mathrm{~Hz}$. When the logging hardware was active, the ignition was turned on. The car was allowed to warm up all the way to operating temperature (185 degrees Fahrenheit) with no external inputs from the driver,

\footnotetext{
${ }^{2}$ The device tested was a preproduction "Version 8" unit on loan from
} CarKnow. 
while the ambient temperature was recorded at the time of starting the ignition. Keeping the vehicle stationary abstracted away potential complications from the measurement, such as engine loading and increased airflow, and allowed a more robust comparison between new and worn engine oil. To eliminate another potential factor, data were logged on days with similar ambient temperatures to reduce variance, while a correction metric was in development.

Initially, old oil was tested. By recording old oil first, then changing to new oil, it was possible to simulate covering significant distance in short time, making testing in similar ambient temperatures much simpler. In this case, the old oil had been used for 9491 miles, or $94 \%$ of useful life according to the in-car estimate.

The first trial involved three cold-to-hot warmups. Then, the oil was changed and the car was driven approximately 10 miles to allow the oil to fully circulate through the engine and properly coat all lubricated surfaces. Once the engine had cooled sufficiently, the new oil was tested three times at similar ambient temperatures to the old oil. After achieving promising results, more samples were collected from controlled warmup cycles to validate the findings at larger scale.

\section{B. Unconstrained Driving}

A second vehicle was tested with old and new oil under uncontrolled, variable engine loading (the vehicles were driven during warmup). This vehicle was driven as normal, imbuing the data with biases from ambient temperature, engine loading, airflow, and passenger load. Parameters identical, as well as different, to those in the controlled experiment were recorded at the same or higher frequency, to provide information about the effect of external factors. As an example of the complexities associated with driving while warming up, a vehicle's engine loading changes substantially under acceleration and heat generation increases, though airflow increases with velocity and therefore provides additional cooling. This vehicle provided richer information to quantify the impact of ambient temperature, impact of throttle-based engine loading and variable passenger loading on the rate of change of coolant temperature. Due to limited time to capture data, this vehicles was not tested under controlled conditions as a baseline.

\section{RESUlts}

Results showed that for the same model vehicle in similar environmental conditions, the engine coolant temperature is an appropriate surrogate for oil viscosity sensing. The unconstrained driving data provided limited utility, as only one vehicle recorded data for processing.

\section{A. Controlled Warmup}

Figure 3 shows a plot of engine coolant temperature versus time for both new and old oil. The plot is aligned so all data start at the same coolant temperature, and increase for a set time window determined to be less than the time at which the engine thermostat opens. In this range, new oil has a shallower slope (reduced $\frac{d T_{\text {cool }}}{d t}$ ) relative to the old oil, and this is repeatable across differing ambient temperatures. This changed slope is because the new oil has reduced relative viscosity and lower internal shear stresses, reducing heat generation.

Engine Coolant Temperature vs. Time

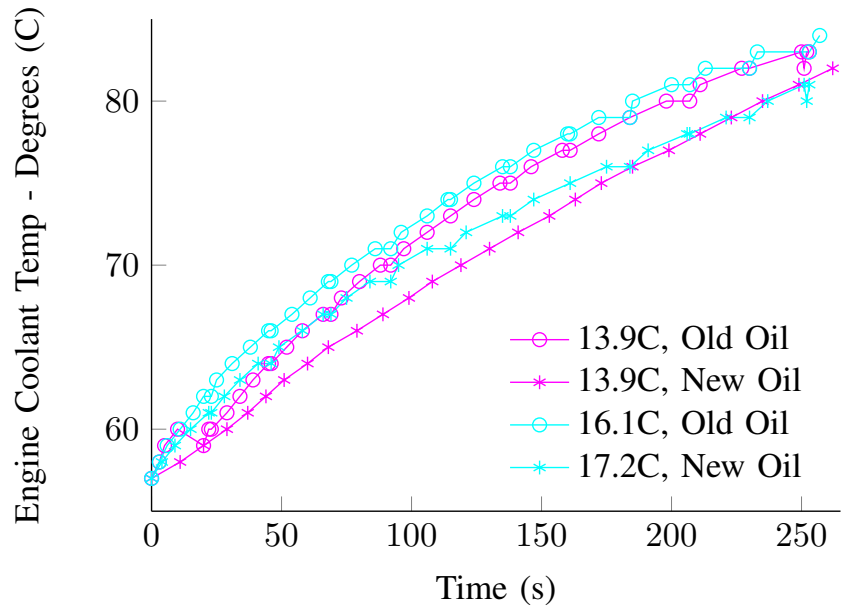

Fig. 3. A plot showing $T_{\text {cool }}\left({ }^{\circ} C\right)$ versus idle time for new and old motor oil at two constant ambient temperatures, highlighting the difference in heat generation for varied oil viscosity. Because the sensor reports with resolution 1C, only switch points (an increase or decrease of 1C) are plotted.

Viewing the data in different context, it is possible to examine the warmup time taken between predefined temperatures and quantitatively demonstrate that the derivative across a temperature range does, for a similar ambient start temperature, relate to the oil life. In Table I, a 2011 Buick Regal was allowed to idle from $65^{\circ} \mathrm{C}$ to $85^{\circ} \mathrm{C}$, a temperature determined to be below the opening point of the vehicle's thermostat.

It is possible to visualize the data more simply in a table format, indicating the time taken to change temperature by $15^{\circ} \mathrm{C}$.

\begin{tabular}{llllllll} 
Sample & $\mathbf{T}_{\mathbf{a m b}}{ }^{\circ} \mathbf{C}$ & $\mathbf{t : ~} \mathbf{6 0 - 6 5}$ & $\mathbf{6 5 - 7 0}$ & $\mathbf{7 0 - 7 5}$ & $\mathbf{7 5 - 8 0}$ & $\mathbf{t}_{\text {total }}$ & $\left({ }^{\circ} \mathbf{C} / \mathbf{s}\right)$ \\
Old - 1 & 12.2 & 44.61 & 43.6 & 51.7 & 73.0 & 212.9 & 0.094 \\
Old - 2 & 13.9 & 42.59 & 36.5 & 47.7 & 64.9 & 191.6 & 0.104 \\
Old - 3 & 16.1 & 28.25 & 39.1 & 47.1 & 61.1 & 175.5 & 0.114 \\
New - 1 & 7.2 & 84.15 & 101.2 & 108.2 & 128.2 & 421.8 & 0.047 \\
New - 2 & 8.9 & 79.14 & 106.2 & 111.2 & 136.2 & 432.8 & 0.046 \\
New - 3 & 13.9 & 78.03 & 102.1 & 108.1 & 124.1 & 412.2 & 0.049 \\
New - 4 & 17.2 & 34.07 & 46.1 & 66.1 & 78.1 & 224.4 & 0.089 \\
New - 5 & 21.1 & 39.07 & 48.1 & 65.1 & 82.2 & 234.5 & 0.085 \\
& TABLE I. & \multicolumn{2}{l}{ TABLE SHOWING CONTROLLED WARMUP } &
\end{tabular}

TIME-TO-TEMPERATURE AND AMBIENT STARTING TEMPERATURE FOR A SINGLE VEHICLE WITH NEW (APPROX. 10 MILE) AND OLD (APPROX. 9500 MILE) OIL.

The table shows that there is a minimal dependence temperature when starting temperatures are not substantially different. There is a clear difference in heat generation between old and new oil, with the older oil heating faster.

\section{B. Unconstrained Driving}

The data collected for unconstrained driving revealed less predictability in rate of heat generation (see Table II). Vi- 


\begin{tabular}{|c|c|c|c|c|c|c|c|c|c|c|c|c|}
\hline Sample & $\mathbf{T}_{\mathbf{a m b}}{ }^{\circ} \mathrm{C}$ & Pass & t: $50-55$ & $55-60$ & $60-65$ & $65-70$ & $70-75$ & $75-80$ & $t_{\text {total }}$ & $\left({ }^{\circ} \mathrm{C} / \mathrm{s}\right)$ & $\mathbf{V}_{\text {avg }}(\mathbf{m p h})$ & RPMavg $_{\text {av }}$ \\
\hline Old - 1 & 21 & 1 & 22.54 & 22.56 & 21.37 & 22.51 & 25.93 & 28.17 & 143.08 & 0.21 & 8.53 & $1156.43^{\circ}$ \\
\hline Old - 2 & 22 & 4 & 20.3 & 17.99 & 14.65 & 9 & 15.78 & 33.81 & 111.53 & 0.27 & 14.43 & 1339.9 \\
\hline Old - 3 & 22 & 2 & 21.39 & 20.25 & 11.24 & 14.62 & 40.52 & 32.67 & 140.68 & 0.21 & 9.9 & 1294.66 \\
\hline Old - 4 & 22 & 2 & 20.3 & 24.79 & 14.64 & 14.62 & 25.89 & 40.54 & 140.78 & 0.21 & 8.36 & 1240.34 \\
\hline Old - 5 & 22 & 1 & 21.99 & 2.02 & 21.01 & 38.14 & 34.07 & 59.05 & 195.28 & 0.15 & 8.93 & 1114.32 \\
\hline Old - 6 & 25 & 1 & 26.67 & 26.68 & 25.52 & 31.32 & 16.24 & 18.56 & 144.99 & 0.21 & 6.66 & 1162.54 \\
\hline Old - 7 & 27 & 2 & 23.65 & 14.65 & 14.63 & 21.41 & 27.02 & 18.01 & 119.37 & 0.25 & 14.97 & 1324.18 \\
\hline Old - 8 & 30 & 2 & 18.03 & 12.37 & 22.53 & 21.4 & 11.25 & 10.16 & 95.75 & 0.31 & 23.24 & 1571.26 \\
\hline New - 1 & 20 & 1 & 24.02 & 27.76 & 22.9 & 12 & 13.02 & 51.06 & 150.77 & 0.2 & 6.54 & 1153.12 \\
\hline New - 2 & 21 & 2 & 18 & 20 & 9.01 & 18 & 23 & 74.01 & 162.03 & 0.19 & 7.34 & 1181.61 \\
\hline New - 3 & 23 & 1 & 22.98 & 23 & 22.01 & 23.02 & 12.99 & 32.05 & 136.05 & 0.22 & 9.07 & 1177.95 \\
\hline New - 4 & 26 & 1 & 25.03 & 17.03 & 16.02 & 21.99 & 21.03 & 14.97 & 116.07 & 0.26 & 17.27 & 1439.92 \\
\hline New -5 & 28 & 2 & 21.02 & 18.01 & 15.98 & 26.01 & 24.03 & 54 & 159.06 & 0.19 & 8.24 & 1107.61 \\
\hline New - 6 & 29 & 2 & 14.15 & 20.12 & 20.01 & 12.97 & 9.01 & 12.99 & 89.25 & 0.34 & 25.51 & 1562.87 \\
\hline
\end{tabular}

TABLE II. TABLE SHOWING UNCONSTRAINED WARMUP TIME-TO-TEMPERATURE FOR A SINGLE VEHICLE WITH NEW (APPROX. 10 MILE) AND OLD (APPROX. 9500 MILE) OIL. AlSO INCLUDED IS ADDITIONAL INFORMATION ABOUT NUMBER OF PASSENGERS (PASS), AVERAGE VEHICLE SPEED ( $V_{a v g}$ ), ENGINE ROTATION $\left(R P M_{a v g}\right)$

sual inspection indicated a substantial impact of engine and passenger loading on engine temperature, to the point where new oil was seen heating up more rapidly than old oil. This result highlights the need for correction factors in any model relying solely on temperature to characterize oil life to minimize the impact of driver style or vehicle loading changes.

Ongoing work is exploring further implementation details for this method of viscosity measurement, including the use of intake air temperature, humidity, barometric pressure and mass airflow rate as correction factors for engine and environmental temperature, vehicle velocity, and engine loading.

\section{CONCLUSION}

From the controlled warmup results, one sees that $\frac{d T_{c o o l}}{d t}$ is an appropriate surrogate metric for oil viscosity and thus may be extended to model remaining oil life when vehicles are stationary during a substantial portion of engine warmup. We have therefore demonstrated the use of an existing, networked sensor present in all OBDII and EOBD equipped vehicle to monitor engine temperature over time and as a result, the state of engine oil.

The model demonstrated in this paper is applicable today for drivers who let their cars idle, for example to heat up in winter or cool down in summer. These analytics may be run in the background and operated transparently for the user.

With more complete information, engine oil intervals may be freed from fixed time and distance intervals, instead being based off of individualized oil metrics that reflect the true condition of the filled fluid. This allows optimal service timing, where the vehicle owner is informed and asked to change engine oil only when necessary thereby minimizing unnecessary costs and improving engine longevity. Users may also be persuaded to take individualized oil data more seriously if they are informed that there is science behind their customtailored recommendation, rather than following a generalized maintenance schedule.

\section{FUTURE WORK}

Future work will show the relationship between rate of coolant temperature change to airflow, engine loading, engine starting temperature, and additional factors such as variable passenger load, so that the same analysis might be done for drivers who get into their vehicles and begin driving immediately. This includes a sensitivity analysis of result dependency on differing environmental conditions and repeatability across vehicle models. Further work on oil viscosity detection aim to reduce the sample capture time such that a short window or instantaneous sample may yield insight into remaining oil life.

\section{REFERENCES}

[1] B. Jakoby, et al. "An automotive engine oil viscosity sensor." IEEE Sensors J. 3.5 (2003): 562-568.

[2] A. Agoston, et al. "Viscosity sensors for engine oil condition monitoring -Application and interpretation of results." Sensors and Actuators A: Physical 121, no. 2 (2005): 327-332.

[3] J. Zhu, et al. "Survey of lubrication oil condition monitoring, diagnostics, and prognostics techniques and systems." Journal of Chemical Science and Technology (2013).

[4] A. Mujahid and F. Dickert. "Monitoring automotive oil degradation: analytical tools and onboard sensing technologies". Anal. and Bioanal. Chem., vol. 404, pp. 1197-1209, September 2012.

[5] M. Mesgarpour, D. Landa-Silva, and I. Dickinson. "Overview of Telematics-Based Prognostics and Health Management Systems for Commercial Vehicles," in Activities of Transport Telematics, ed. J. Mikulski, pp. 123-130. Springer Berlin Heidelberg, 2013.

[6] Title 13, California Code Regulations, Section 1968.2, Malfunction and Diagnostic System Requirements for 2004 and Subsequent Model-Year Passenger Cars, Light-Duty Trucks, and Medium-Duty Vehicles and Engines (OBDII). 2013.

[7] Greening, Paul. "European vehicle emission legislation — present and future." Topics in Catalysis 16, no. 1-4 (2001): 5-13.

[8] S. Kumar, J. Paefgen, E. Wilhelm and S. Sarma, "Integrating on-board diagnostics speed data with sparse GPS measurements for vehicle trajectory estimation". SICE Annual Conference (SICE), 2013 Proceedings of, pp. 2302-2308, September 2013.

[9] J. Siegel. "Design, development, and validation of a remotely reconfigurable vehicle telemetry system for consumer and government applications." S.M. thesis, Mech. Eng., MIT, Cambridge, MA, 2013. * On hold at MIT library; contact author for details.

[10] Auterra: "Dash Dyno SPD" http://www.auterraweb.com/dashdynoseries.html. 